\section{Pathogenesis of Atherosclerosis A Review}

\section{Abstract}

In this review, we would discuss the chief pathways involved in the pathophysiology of atherosclerosis. We would also highlight the end terminal events of this sequel with due consideration to risk factors, clinical features, diagnosis and treatment. The aim of the review is to get into the fine details of all possible causes and pathophysiologic mechanisms responsible for atherosclerosis so that new treatment modalities can emerge and reduce the morbidity and mortality associated with atherosclerosis.

Received: August 05, 2016; Accepted: August 22, 2016; Published: August 29, 2016

\section{Introduction}

Atherosclerosis has been derived from a Greek word, Athero meaning gruel [1]. Marchand introduced the term "atherosclerosis" describing the assosciation of fatty degeneration and vessel stiffening [2]. It's the patchy intramural thickening of the subintima. The earliest lesion is the fatty streak. Fatty streak evolve to fibrous plaque and unstable plaque are responsible for clinical events.

Atherosclerosis is marked by atheromas, patchy intimal plaques. Most common location is lumen of medium sized and large arteries. The plaque has cellular component -namely of inflammatory cells, smooth muscle cells, a fibrous component of -connective tissue and a fat component of lipids. Prominent risk factors of consideration are Hypertension, Diabetes, Dyslipidemia, obesity, sedentary life style, Family history, smoking. Intraplaque rupture, bleeding, thrombosis and stenosis cause symptoms. Diagnosis is clinical and definitive diagnosis is made through Imaging tests. Management plan includes behavior modifications (Physical activity with low caloric diet, rich in fiber component) and main class of drugs used in treatment are antiplatelet drugs and antiatherogenic drugs.

It is the leading cause of morbidity and mortality in the US and western world. In the current era cardiovascular disease (CVD) remains the MCC of death all over the world. In 2008, 17 million deaths were recorded from CVD. More than 3 million of these deaths occurred in people below the age of 60 and could have largely been prevented [3]. There are growing inequalities in the occurrence and outcome of CVD between countries and social classes.

\section{Discussion}

\section{Aziz $\mathrm{M}^{1}$ and Yadav KS² \\ Pri-Med Care, Lewisville, Texas 75067, USA \\ 2 Principal, RGCP, Bhopal, India}

\section{Corresponding author: Maria Aziz}

Director, Pri-Med Care, Lewisville, Texas 75067, USA.

mariz1979@yahoo.in

Tel: 9723656942

Citation: Aziz M, Yadav KS. Pathogenesis of Atherosclerosis. Med Clin Rev. 2016, 2:3.

We would discuss the chief pathways involved in the pathophysiology of atherosclerosis. We would also highlight the end terminal events of this sequel with due consideration to risk factors, clinical features, diagnosis and treatment.

\title{
Pathophysiology
}

Atherosclerosis is a chronic inflammatory disease. Atherosclerosis begins with fatty streak which is a accumulation of lipid laden foam cells in the intimal layer of the artery [4]. Lipid retention is the first step in the pathogenesis of atherosclerosis which is followed by chronic inflammation at susceptible sites in the walls of the major arteries lead to fatty streaks, which then progress to fibroatheromas which are fibrous in nature (Table 1) [5,6].

Atherosclerosis is a continuous progressive development. Fatty streak develop at $11-12$ years and fibrous plaques at $15-30$ years (Figure 1, depicts the conversion of Fatty Streak to Fibrous Plaques) [7] and they develop at the same anatomic sites as the fatty streaks making it more evident that fibrous plaques arise from fatty streak. Pathologic intimal thickening leads to fatty streak, leads to fibrous cap atheromas, lead to plaques, finally leading to sudden cardiac death $[8,9]$.

Fatty streaks evolve to atherosclerotic plaques which is composed of three components namely of inflammatory cells, smooth muscle cells, a fibrous component of-connective tissue and a Fat component of lipids [10].

Endothelial Injury plays the inciting role. Turbulent blood flow leads to endothelial dysfunction, it inhibits production of NO, 
Table 1 Stages of Atherosclerosis: Modified AHA consensus classification based on morphologic descriptions.

\begin{tabular}{|c|c|c|}
\hline & Description & Thrombosis \\
\hline \multicolumn{3}{|c|}{ Nonatherosclerotic intimal lesions } \\
\hline Intimal thickening & $\begin{array}{l}\text { Normal accumulation of smooth muscle cells (SMCs) in the intima in the absence of lipid } \\
\text { or macrophage foam cells. }\end{array}$ & Absent \\
\hline Intimal xanthoma & $\begin{array}{l}\text { Superficial accumulation of foam cells without a necrotic core or fibrous cap; based on } \\
\text { animal and human data, such lesions usually regress. }\end{array}$ & Absent \\
\hline \multicolumn{3}{|c|}{ Progressive atherosclerotic lesions } \\
\hline Pathologic intimal thickening & SMC-rich plaque with proteoglycan matrix and focal accumulation of extracellular lipid & Absent \\
\hline Fibrous cap atheroma & $\begin{array}{l}\text { Early necrosis: focal macrophage infiltration into areas of lipid pools with an overlying } \\
\text { fibrous cap Late necrosis: loss of matrix and extensive cellular debris with an overlying } \\
\text { fibrous cap. }\end{array}$ & Absent \\
\hline Thin cap fibroatheroma & $\begin{array}{l}\text { A thin, fibrous cap }(<65 \mu \mathrm{m}) \text { infiltrated by macrophages and lymphocytes with rare or } \\
\text { absence of SMCs and a relatively large underlying necrotic core; intraplaque hemorrhage/ } \\
\text { fibrin may be present. }\end{array}$ & Absent \\
\hline \multicolumn{3}{|c|}{ Lesions with acute thrombi } \\
\hline Plaque rupture & $\begin{array}{l}\text { Fibroatheroma with fibrous cap disruption; the luminal thrombus communicates with the } \\
\text { underlying necrotic core }\end{array}$ & $\begin{array}{l}\text { Occlusive or } \\
\text { nonocclusive }\end{array}$ \\
\hline Plaque erosion & $\begin{array}{l}\text { Plaque composition, as above; no communication of the thrombus with the necrotic core; } \\
\text { can occur on a plaque substrate of pathologic intimal thickening or fibroatheroma }\end{array}$ & Usually nonocclusive \\
\hline Calcified nodule & $\begin{array}{l}\text { Eruptive (shedding) of calcified nodules with an underlying fibrocalcific plaque with } \\
\text { minimal or absence of necrosis }\end{array}$ & Usually nonocclusive \\
\hline \multicolumn{3}{|c|}{ Lesions with healed thrombi } \\
\hline $\begin{array}{l}\text { Fibrotic (without calcification) } \\
\text { Fibrocalcific (+/- necrotic core) }\end{array}$ & $\begin{array}{l}\text { Collagen-rich plaque with significant luminal stenosis; lesions may contain large areas } \\
\text { of calcification with few inflammatory cells and minimal or absence of necrosis; these } \\
\text { lesions may represent healed erosions or ruptures }\end{array}$ & Absent \\
\hline
\end{tabular}

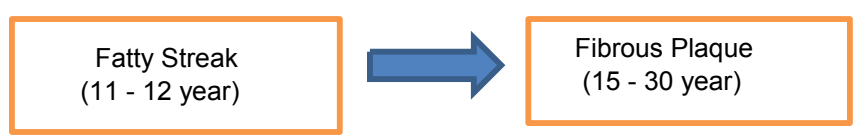

Figure 1 Conversion of fatty streak to Fibrous plaques.

a potent vasodilator and stimulates production of adhesion molecules which attract inflammatory cells. Other risk factors also contribute to this step. The net result is Monocytes and T cells bind to the endothelial cells and migrate to the subendothelial space. Lipids in the blood, LDL, VLDL bind to endothelial cells and oxidize in the subendothelial space. Monocytes in subendothelial space engulf oxidized LDL and transform to foam cells. This mark the first stage i.e., fatty streak. Macrophages further elaborate proinflammatory cytokines which recruit smooth muscle cells. There is smooth muscle cell replication and increase in dense extracellular matrix. End result lesion is a subendothelial fibrous plaque composed of lipid core surrounded by smooth muscle cells and connective tissue fibers (Figures 2 and 3) [11].

There is sequential involvement of arterial layers, intima, then media and finally adventitia. Arterial wall lesions have a central cholesterol rich lipid core surrounded by inflammatory response. Every lesion has lipid accumulation and inflammation. Plaque distorts media/adventitia, increases caliber of arterial lumen and decreases its size simultaneously. New Vasa Vasorum invade diseased intima, cause hemorrhage within arterial wall, leading to intramural hemorrhage and increased fibrous tissue. Rupture of thin fibrous caps leads to thrombosis and healing. Cyclic healing of clinically silent ruptures leads to multiple layer of healed tissue and the end result is sudden cardiac death. Calcium deposits as small aggregates convert later to large nodules in the wall. Erosion of endothelium leads to thrombosis. Increase plaque mass causes stenosis and finally leading to lethal ischemia [12].

There are two types of plaques stable and unstable [13]. Stable plaques regress or are static or they grow slowly. Unstables plaques are complicated by erosion, fissure, rupture and cause stenosis, thrombosis, infarction. Most clinical events result from complications of unstable plaque, hence plaque stabilization can reduce morbidity and mortality associated with atherosclerosis.

Plaque is ruptured by enzymes secreted by activated macrophages in the plaque. Once plaque ruptures, plaque contents get exposed to circulating blood and the end result is thrombosis [14]. The resultant thrombosis may change plaque shape, occlude lumen of blood vessel, may embolise or the plaque contents may embolise. Low risk plaques are more fibrous in content and have low lipids and do not cause $100 \%$ blockade while unstable plaques have thick lipid core and thin fibrous cap narrow lumen $<50 \%$ and tend to rupture unpredictably [15].

The end result of the stenosis caused by the plaques are the Terminal Events-Acute Coronary Syndrome, Myocardial Infarction, Fatal Arrhythmias, Sudden Cardiac death (Figures 4 and 5 depict the terminal events that arise due to stenosis due to plaques) [16].

\section{Risk factors}

Age, Family history, Male sex, smoking, Diabetes mellitus, hypertension, alcohol, Chlamydia infection, Hyper homocysteinemia, Obesity, Sedentary lifestyle [17]. 
Lipid Retention

Oxidation Modification

$\downarrow$

Chronic Inflammation

At Susceptible Sites in walls of major

Arteries

$\downarrow$

Initial Fatty Streak

$\downarrow$

Fibrous plaques

$\downarrow$

Vulnerable to Rupture

$\downarrow$

Thromosis/Stenosis

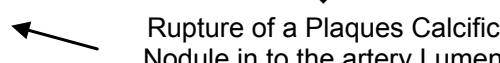

Traditional Rise Factors

$\oplus$ 1. Hypertension

2. Diabetes Mellitus

3. Smoking

4. Obesity

5. Genetics

(Early Childhood Adolescence)

Erosion of the Surfaces of Some Plaques $\downarrow$

Nodule in to the artery Lumen

Figure 2 Stages of Atherosclerosis.

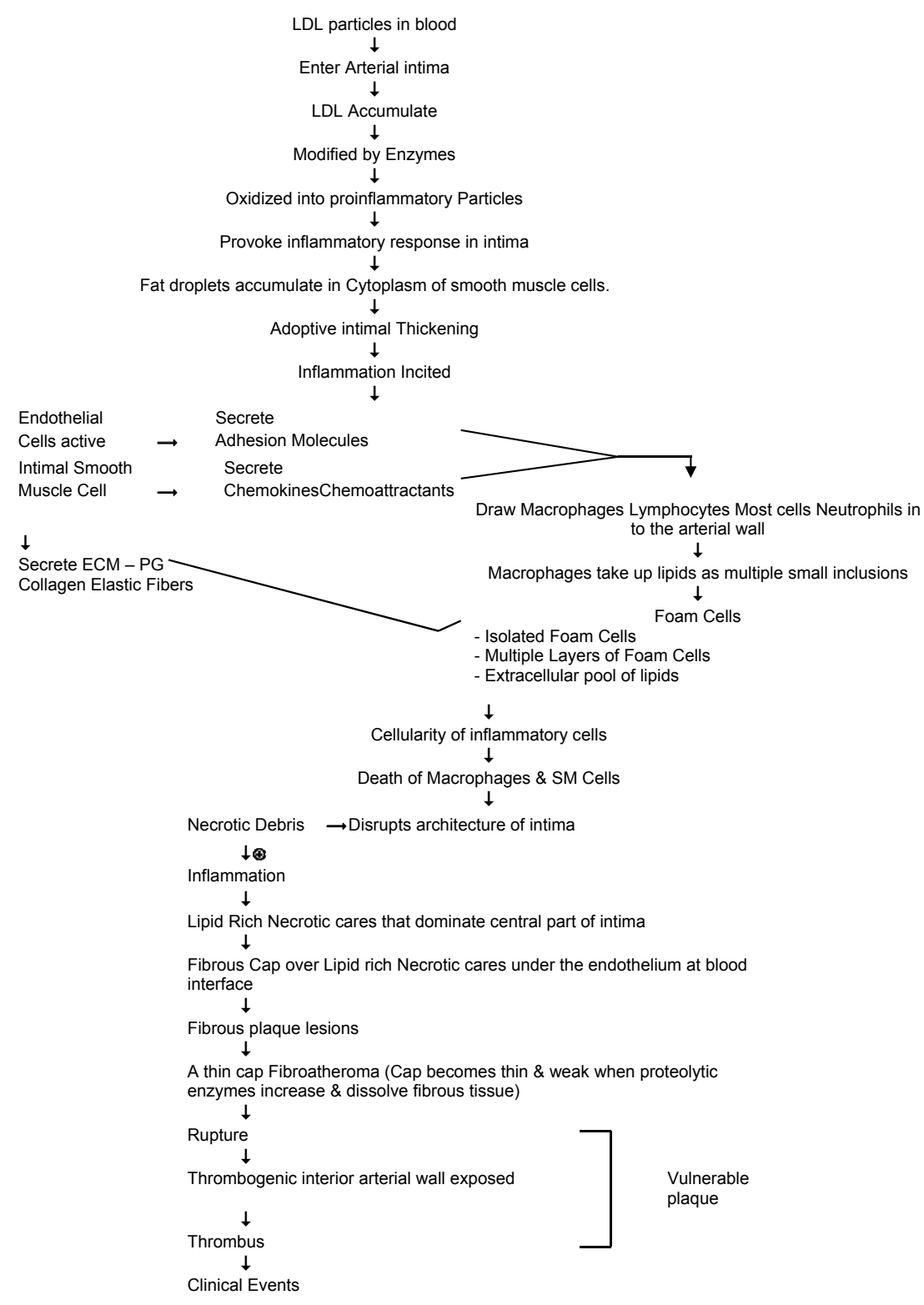

Figure 3 Pathway of Atherosclerosis. 


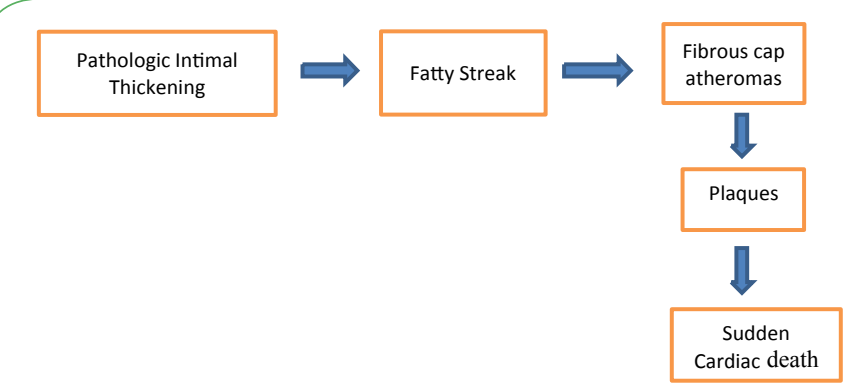

Figure 4

Steps in terminal events.

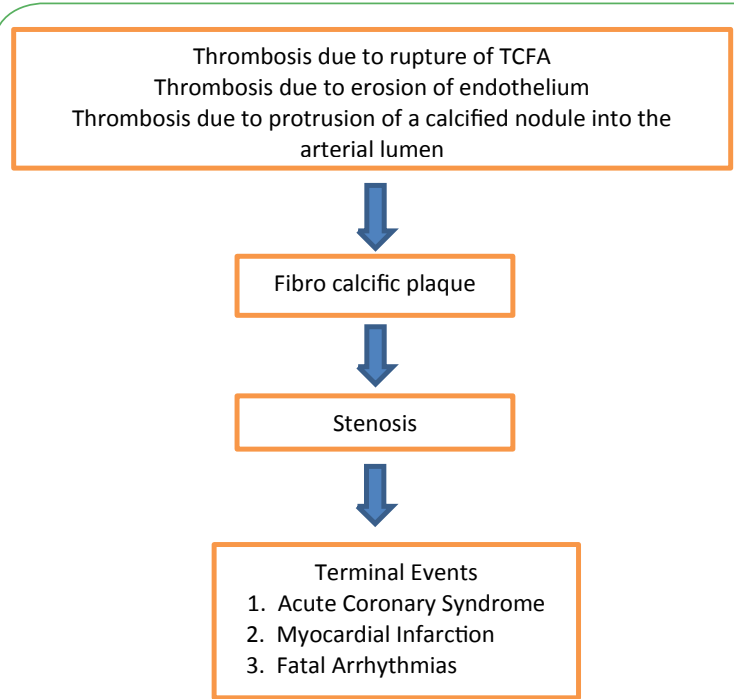

Figure 5 Terminal events that arise due to stenosis due to Plaques.

\section{Location}

Elastic and muscular arteries. MC arteries affected are aorta, carotid, coronary and ileofemoral arteries. MC artery to be involved is Aorta. Branch points are the common sites. Proximal coronary arteries are more susceptible [18].

\section{Clinical features}

Atherosclerosis is initially asymptomatic [19]. Symptoms develop when lesions impede blood flow. When plaques grow, arterial lumen is reduced causing transient ischemic symptoms, stable exertional angina, intermittent claudication, unstable angina, infarction, ischemic stroke, rest pain in the limbs, aneurysm, arterial dissection, sudden death [20].

\section{Diagnosis}

Patients with signs and symptoms of ischemia should be evaluated using history, physical examination, fasting lipid profile, plasma glucose and $\mathrm{HbA1c}$. Patients with documented disease at one site should be evaluated at other sites.

CT Angiography, is often used as an initial screening test [21]. Other diagnostic procedures used are catheter based tests-intravascular ultrasonography, angioscopy, plaque thermography, elastography, immunoscintigraphy. Certain serum inflammatory markers CRP, LP associated phospholipase A2 predict cardiovascular events. Other Imaging studies that detect plaque are-Angiography, USG, CIMT, MRI.

\section{Treatment}

Behavior Modifications include a diet rich in fruits, vegetables, fibers with regular physical activity and smoking cessation could help in getting a favourable lipid profile. Drugs to treat Dyslipidemia, hypertension and diabetes are often required. These help in improving endothelial function, reducing inflammation and give a favourable clinical outcome. Additional drugs used are statins, antiplatelet drugs (aspirin, clopidogrel), ACE inhibitors and Beta Blockers. Aspirin is indicated for prevention of coronary atherosclerosis in high risk patients. Clopidogrel is used for patients who are intolerant to aspirin and also for treating ST segment and non ST segment elevation MI. Statins, ACE inhibitors, ARB reduce risk of atherosclerosis with their anti-inflammatory properties. Statins by stabilizing the plaques, play vital role in management of atherosclerosis.

Statins induce changes in plaque tissues, hence are important in causing regression in atherosclerosis (Table 2 discusses the Statins induced changes in plaque tissues) $[4,11,18]$.

Fish oil supplements-Omega 3 fatty acids play vital role in creating favourable lipid profile. Vitamins-Folate, vitamin B6 and B 12 treat hyper homocysteinemia which is an important cause of Dyslipidemia.

Table 2: Statins induced changes in Plaque tissues.

1. Alter Plaque Size
2. Alter Cellular Composition/Chemical Composition.
3. Alter Arterial Lesions.
4. Reduce Clinical Consequences/Events.
5. Alter Plaque Chemical Composition.
6. Alter Plaque biological activities centered on inflammation +
$\quad$ Cholesterol
7. Decrease risk of clinical events.
8. $\downarrow$ LDL \& $\uparrow$ HDL
9. $\downarrow$ CVS morbidity/Mortality
10. Retard development of atherosclerotic plaque \& cause plaque
stabilization.
11. $\downarrow$ Rate of Plaque development
12. $\downarrow$ Coronary plaque progression.
13. $\downarrow$ atheroma volume.
14. $\downarrow$ Macrophage in Plaque
$\downarrow$ Lymphocyte in Plaque
$\downarrow$ Lipid content
$\downarrow$ Collagen content
$\downarrow$ Inflammation
$\downarrow$ Oxidation
$\quad \downarrow$ Enzyme Proteolytic activity
$\quad($ MMP-2/MMP-9/COX-2)
$\downarrow$ Rupture
15. $\downarrow$ Development of atherosclerotic plaques.
16. Clinical events delayed.
17. Favorable Alteration in composition of advanced plaque.
18. $\downarrow$ Plaque size.
19. $\downarrow$ CVD events/ $\downarrow$ Clinical Consequences.
20. Alter Arterial Lesion.




\section{Conclusion}

Atherosclerosis is the leading cause of death in the developed countries. Deep understanding of the causes and underlying mechanism of pathogenesis will help to delineate causes and will help to plan out innovative management. As our knowledge about the pathogenesis of atherosclerosis improves more treatment options will emerge. 


\section{References}

1 Virmani R, Burke AP, Farb A, Kolodgie FD (2006) Pathology of the vulnerable plaque. J Am Coll Cardiol 47: C13-C18.

2 Aschoff L (1933) Introduction. In: Cowdry EV (ed.) Arteriosclerosis: A Survey of the Problem. Macmillan, New York.

3 Beaglehole R, Magnus P (2002) The search for new risk factors for coronary heart disease: occupational therapy for epidemiologists? Int J Epidemiol 31: 1117-1122.

4 Alexander RW, Dzau VJ (2000) Vascular biology: the past 50 years. Circulation 102: IV-112-IV-116.

5 Pedrigi RM, de Silva R, Bovens SM, Mehta VV, Petretto E, et al. (2014) Thin-cap fibroatheroma rupture is associated with a fine interplay of sand wall stress. Arterioscler Thromb Vasc Biol 34: 2224-2231.

6 Collins R, Armitage J, Parish S, Sleigh P, Peto (2003) R: MRC/BHF Heart Protection Study of cholesterol-lowering with simvastatin in 5963 people with diabetes: a randomised placebo-controlled trial. Lancet 361: 2005-2016.

7 Libby P (2002) Inflammation in atherosclerosis. Nature 420: 868-874.

8 Meijer WT, Hoes AW, Rutgers D, Bots ML, Hofman A, et al. (1998) Peripheral arterial disease in the elderly: the Rotterdam Study. Arterioscler Thromb Vasc Biol 18: 185-192.

9 Simoons L, Saelman M (1994) Effect of simvastatin on coronary atheroma: the Multicentre Anti-Atheroma Study (MAAS). Lancet 344: 633-638.

10 Ridker PM, Danielson E, Fonseca FA, Genest J, Gotto AM, et al. (2009) Reduction in C-reactive protein and LDL cholesterol and cardiovascular event rates after initiation of rosuvastatin: a prospective study of the JUPITER trial. Lancet 373: 1175-1182.

11 Fowkes FG, Housley E, Riemersma RA, Macintyre CC, Cawood $\mathrm{EH}$, et al. (1992) Smoking, lipids, glucose intolerance, and blood pressure as risk factors for peripheral atherosclerosis compared with ischemic heart disease in the Edinburgh Artery Study. Am J Epidemiol 135: 331-340.
12 Scott G, Diane B, Luther C, Richard C, Margo D, et al. (2001) Executive Summary of The Third Report of The National Cholesterol Education Program (NCEP) Expert Panel on Detection, Evaluation, and Treatment of High Blood Cholesterol In Adults (Adult Treatment Panel III). JAMA 285: 2486-2497.

13 Ingolfsson IO, Sigurdsson G, Sigvaldason $\mathrm{H}$, Thorgeirsson G, Sigfusson $\mathrm{N}$, et al. (1994) A marked decline in the prevalence and incidence of intermittent claudication in Icelandic men 1968-1986: a strong relationship to smoking and serum cholesterol: the Reykjavik Study. J Clin Epidemiol 47: 1237-1243.

14 Jonason T, Bergstrom R (1987) Cessation of smoking in patients with intermittent claudication: effects on the risk of peripheral vascular complications, myocardial infarction and mortality. Acta Med Scand 221: $253-260$.

15 Rodriguez-Granillo GA, Agostoni P, Garcia-Garcia HM, Biondi-Zoccai GG, McFadden E (2007) Meta-analysis of the studies assessing temporal changes in coronary plaque volume using intravascular ultrasound. Am J Cardiol 99: 5-10.

16 Naghavi M, Libby P, Falk E, Casscells SW, Litovsky S, et al. (2003) From vulnerable plaque to vulnerable patient a call for new definitions and risk assessment strategies: part I. Circulation 108: 1664-1672.

17 Quick CR, Cotton LT (1982) The measured effect of stopping smoking on intermittent claudication. Br J Surg 69: S24-S26.

18 Silva Marques J, Pinto FJ (2014) The vulnerable plaque: Current concepts and future perspectives on coronary morphology, composition and wall stress imaging. Rev Port Cardiol 33: 101-110.

19 Law M, Tang JL (1995) An analysis of the effectiveness of interventions intended to help people stop smoking. Arch Intern Med 155: 1933-1941.

20 Cannon CP, Braunwald E, McCabe CH, Rader DJ, Rouleau JL, et al. (2004) Intensive versus moderate lipid lowering with statins after acute coronary syndromes. N Engl J Med 350: 1495-1504.

21 Rosamond W, Flegal K, Furie K, Go A, Greenlund K, Haase N, et al. (2008) Heart disease and stroke statistics-2008 update: a report from the American Heart Association Statistics Committee and Stroke Statistics Subcommittee. Circulation 117: e25-e146. 